Check for updates

Cite this: Mater. Adv., 2021, 2, 7709

Received 6th September 2021, Accepted 25th September 2021

DOI: 10.1039/d1ma00811k

rsc.li/materials-advances

\section{Effect of bulky 2,6-bis(spirocyclohexyl)- substituted piperidine rings in bis(hindered amino)trisulfide on thermal healability of polymethacrylate networks $\dagger$}

\author{
Motohiro Aiba, (D) *a Take-aki Koizumi, (DD ${ }^{b}$ Kazuaki Okamoto, ${ }^{a}$ Motoshi Yamanaka, ${ }^{a}$ \\ Michinari Futamura, ${ }^{a}$ Yuzo Ishigaki, ${ }^{a}$ Mitsuo Oda, ${ }^{a}$ Chihiro Ooka, ${ }^{a}$ \\ Akira Takahashi (D) ${ }^{c}$ and Hideyuki Otsuka (D) ${ }^{\text {cd }}$
}

\begin{abstract}
This paper describes the synthesis of highly sterically hindered piperidinyl trisulfide with four spirocyclohexyl moieties, bis(2,6-bis[spirocyclohexyl]piperidine-1-yl)trisulfide (BIBSCPS-S3), from commercially available starting materials in short steps and its application as a dynamic covalent bond for thermally healable polymer networks. Conformational study on the BIBSCPS-S3 moiety in the solid state is performed by single-crystal X-ray diffraction. In bulk, a stress-relaxation experiment reveals that the increase in steric hindrance can not only decrease the activation energy for thermal exchange reactions but also suppress chain-transfer reactions during radical polymerization to some extent. Therefore, the dynamic cross-linking point containing BIBSCPS-S3 moiety can be efficiently incorporated into polymer networks with ethyl, $n$-butyl, or $n$-hexyl methacrylate monomers, which is in good accordance with the relatively low chain-transfer constants of the BIBSCPS-S3 moiety determined by the Mayo equation. As a result, BIBSCPS-S3-cross-linked poly(n-hexyl methacrylate) exhibits nearly quantitative damage healability only by simple hot pressing at $90{ }^{\circ} \mathrm{C}$ under mild pressure for $24 \mathrm{~h}$.
\end{abstract}

\section{Introduction}

Dynamic covalent bonds (DCBs), which can realize selective dissociation, reformation, or exchange under certain external stimuli, have attracted considerable attention. ${ }^{1}$ Since DCBs can reorganize the macromolecular architectures or compositions, they can produce a macroscopic functional response (such as shape-memory properties, self-healing properties, reprocessability, and so on) to the environment and surrounding stimuli. ${ }^{2-5}$ Such macroscopic responses are strongly influenced by the nature of DCBs; therefore the macroscopic dynamic properties can be controlled by careful selection of the DCBs. Although various kinds of DCBs have been reported, many of

\footnotetext{
${ }^{a}$ Nagoya Municipal Industrial Research Institute, 3-4-41 Rokuban, Atsuta-ku, Nagoya 456-0058, Japan. E-mail: aiba.motohiro@nmiri.city.nagoya.jp

${ }^{b}$ Advanced Instrumental Analysis Center, Shizuoka Institute of Science and Technology, 2200-2 Toyosawa, Fukuroi, Shizuoka 437-8555, Japan ${ }^{c}$ Department of Organic and Polymeric Materials, Tokyo Institute of Technology, 2-12-1 Ookayama, Meguro-ku, Tokyo, 152-8550, Japan

${ }^{d}$ Department of Chemical Science and Engineering, Tokyo Institute of Technology, 2-12-1 Ookayama, Meguro-ku, Tokyo, 152-8550, Japan

$\dagger$ Electronic supplementary information (ESI) available. CCDC 2098390. For ESI and crystallographic data in CIF or other electronic format see DOI: 10.1039/ d1ma00811k
}

them require high temperature or specific catalysts to exert their dynamic nature. ${ }^{6,7}$ Thus, further development of the library of DCBs triggered by readily usable stimuli (i.e. moderate heat and visible light) has been required to realize more facile use of the dynamic functions.

Bis(dialkylamino) disulfides were found to exhibit lower bond dissociation energy of S-S linkage (e.g., BDE of 26-31 kcal mol $\left.{ }^{-1}\right)$ than dialkyldisulfides $\left(60-70 \mathrm{kcal} \mathrm{mol}^{-1}\right)$, since the generated thiyl radicals are stabilized by the resonance from the lone pair of electrons on the nitrogen atoms. ${ }^{8}$ Nevertheless, bis(dialkylamino) disulfides are rarely studied in organic chemistry, and the respective polymers are very much in their infancy. In 2016, Takahashi et al. developed a simple method to synthesize a sterically hindered bis(dialkylamino) disulfide, bis(2,2,6,6-tetramethylpiperidin-1yl)disulfide (BITEMPS-S2). ${ }^{9}$ The BITEMPS-S2 unit behaves as a stable covalently bonded structure at room temperature and exhibits reversible homolytic dissociation of the S-S linkage above $80{ }^{\circ} \mathrm{C}$ (as shown in Scheme 1a). These dissociated thiyl radicals from the BITEMPS-S2 unit were found to be inert and highly stable in air even at increased temperatures. By virtue of the unique dynamic behavior, BITEMPS-S2 was utilized to impart the specific features such as self-healing and reprocessable cross-linked polymers, the fusion of different cross-linked 


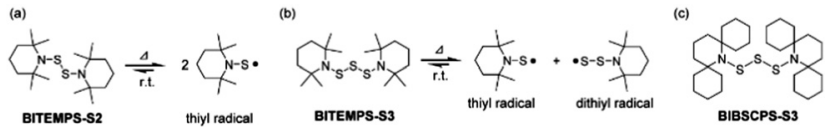

Scheme 1 Characteristic dynamic behaviors of (a) BITEMPS-S2 and (b) BITEMPS-S3 moieties. (c) Chemical structure of a BIBSCPS-S3 moiety.

polymers, the topological rearrangement from linear to cyclic polymers, and self-strengthening of cross-linked elastomers. ${ }^{10-16}$ Afterward, we reported that the trisulfide analogue of BITEMPS-S2, bis(2,2,6,6-tetramethylpiperidin-1-yl)trisulfide (BITEMPS-S3), also behaves as a DCB to produce air-stable thiyl and dithiyl radicals upon heating at mild temperature (as shown in Scheme 1b). ${ }^{17}$ Additionally, the poly(n-hexyl methacrylate) network containing BITEMPS-S3 units at cross-linking points afforded nearly quantitative damage healability only by simple hot pressing at $110{ }^{\circ} \mathrm{C}$ for $24 \mathrm{~h}$, which were almost comparable to one containing BITEMPS-S2 units $\left(100{ }^{\circ} \mathrm{C}\right.$ for $\left.24 \mathrm{~h}\right)$. Although these results indicate that not only bis(dialkylamino) disulfide but also bis(dialkylamino) trisulfide should be recognized as one of the intrinsic DCBs, it is necessary to develop DCBs triggered at more moderate temperature.

Besides, it is well known that the introduction of steric hindrance can lower the BDE. For example, Jing et al. reported that the sterically highly hindered alkoxyamines containing two six-membered rings at the 2,6-position in a piperidine ring turned out to become an efficient initiator for nitroxidemediated polymerization even at $50{ }^{\circ} \mathrm{C}$ due to its lower BDEs of $\mathrm{C}-\mathrm{O}$ linkages. ${ }^{18}$ Although various kinds of sterically hindered nitroxides were synthesized, the effect of partial modification to piperidine rings in BITEMPS-S2 and BITEMPS-S3 units still remains unclear. ${ }^{18-26}$

Herein, we report the dynamic covalent chemistry of bis(2,6bis[spirocyclohexyl]piperidin-1-yl)trisulfide (BIBSCPS-S3), where the bis(dialkylamino) trisulfide moiety is sterically hindered by two spirocyclohexyl groups in each piperidine ring, and describe its intrinsic self-healing property in the polymer networks.

\section{Results and discussion}

\section{Preparation, molecular structure, and disproportionation}

BIBSCPS-S3-OH was prepared from the commercially available compound, 2,2,6,6-tetramethylpiperidin-4-one, in five steps according to Scheme 2. By applying a previously reported procedure by Yamada et al. about the effective 2,6-substitution of piperidine derivatives via crossed aldol condensation, the

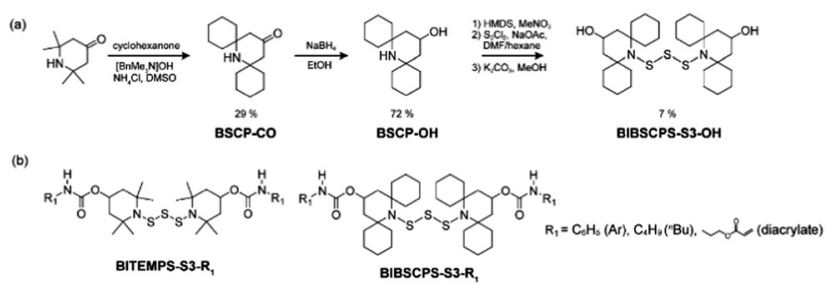

Scheme 2 (a) Synthesis of BIBSCPS-S3-OH. (b) Chemical structure of BITEMPS-S3 or BIBSCPS-S3 derivatives. 2,6-position of the 2,2,6,6-tetramethylpiperidin-4-one was successfully substituted by two spirocyclohexyl groups to afford the 2,6-bis(spirocyclohexyl)-4-piperidone (BSCP-CO) using $\mathrm{NH}_{4} \mathrm{Cl}$ and Triton B. ${ }^{22}$ The reduction of BSCP-CO with $\mathrm{NaBH}_{4}$ afforded the corresponding alcohol compound, 4-hydroxy-2,6-bis(spirocyclohexyl)piperidone (BSCP-OH). Subsequently, the reaction of the TMS-protected BSCP-OH, 4-tetramethylsiloxy-2,6-bis(spirocyclohexyl)piperidine, with disulfur dichloride $\left(\mathrm{S}_{2} \mathrm{Cl}_{2}\right)$ was performed in a DMF-hexane mixture and gave trisulfide and tetrasulfide mixtures, indicating that it would be difficult to provide a disulfide linkage due to a steric inhibition during reaction. After deprotection of the reaction mixture, BIBSCPS-S3-OH was easily separated from its tetrasulfide analogue due to the solubility difference in ethyl acetate and its molecular structure was confirmed by ${ }^{1} \mathrm{H} \mathrm{NMR}$, ${ }^{13} \mathrm{C}$ NMR, and Fourier transform-infrared (FT-IR) spectra and mass spectroscopy (see the Experimental Section in the ESI $\dagger$ ).

First of all, the molecular structure of BIBSCPS-S3-OH was investigated using X-ray crystal analysis by recrystallization. For BIBSCPS-S3-OH, four spirocyclohexyl groups at the 2,6-position of two piperidine moieties exist in a chair conformation. It is noteworthy that all spirocyclohexyl groups are away from the $\mathrm{N}-\mathrm{S}$ bonds as shown in Fig. 1. According to a previous report by Yamamoto et al., the replacement of a hydrogen atom with a methyl group in the amine group of the 2,6-bis(spirocyclohexyl)piperidine, where one spirocyclohexyl ring is away from and the other is toward the amine group, increased the steric repulsions from the $\mathrm{N}-\mathrm{CH}_{3}$ group so that both spirocyclohexyl rings exhibited the same conformation to be away from the $\mathrm{N}-\mathrm{CH}_{3}$ group. ${ }^{23}$ Along the same line, the reason why spirocyclohexyl groups sit in a chair conformation away from the $\mathrm{N}-\mathrm{S}$ bonds would be due to decreasing the steric hindrance from sulfur atoms and the cyclohexyl hydrogen atoms.

Then, the thermal bond dissociation behavior of the BIBSCPS-S3 moiety was studied. As has been previously reported, the trisulfide linkage in the BITEMPS-S3 moiety as a DCB disproportionated to the corresponding disulfide and tetrasulfide linkages because unsymmetrical and homolytic bond cleavage of the trisulfide linkage affords both thiyl and dithiyl radicals. ${ }^{17}$ Therefore, to investigate the thermal disproportionation reaction of the BIBSCPS-S3 moiety, the phenyl urethanated derivative (BIBSCPS-S3-Ar) was prepared due to the poor solubility of BIBSCPS-S3-OH. As shown in Fig. S3 (ESI $\dagger$ ),

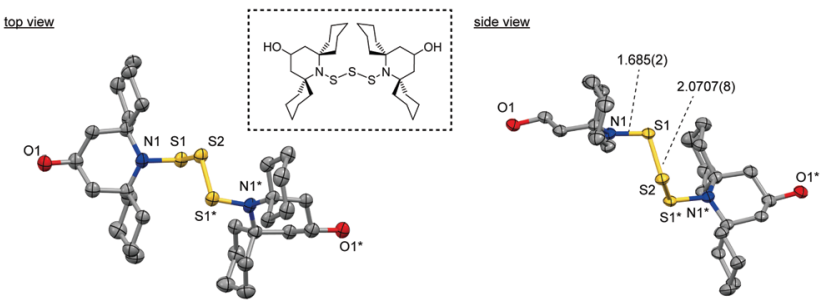

Fig. 1 X-Ray crystal structures of BIBSCPS-S3-OH. The hydrogen atoms are omitted for clarity. The selected angles $\left({ }^{\circ}\right)$ and torsions $\left({ }^{\circ}\right)$; BIBSCPS-S3$\mathrm{OH}$; N1-S1-S2:106.53(8), S1-S2-S1*: 112.9(6), N1-S1-S2-S1*: 93.64(16). Inlet chemical structure indicates conformation of spirocyclohexyl groups away from $\mathrm{N}-\mathrm{S}$ bonds. 

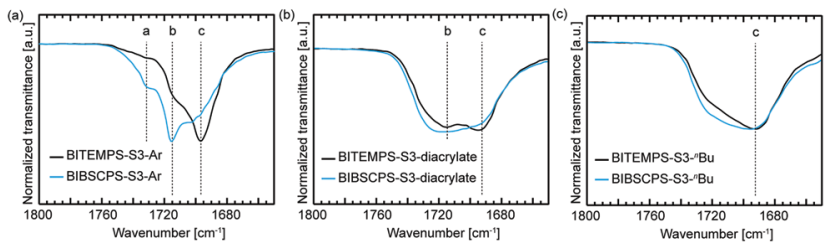

Fig. 2 FT-IR spectra of (a) BITEMPS-S3-Ar and BIBSCPS-S3-Ar, (b) BITEMPS-S3-diacrylate and BIBSCPS-S3-diacrylate, and (c) BITEMPS-S3- ${ }^{n} \mathrm{Bu}$ and BIBSCPS-S3- ${ }^{n} \mathrm{Bu}$. The dotted lines " $\mathrm{a}, \mathrm{b}, \mathrm{C}$ " represent the peaks attributed to the $-\mathrm{C}=\mathrm{O}$ stretching vibration of free $\left(1732 \mathrm{~cm}^{-1}\right)$, disordered $\left(1715 \mathrm{~cm}^{-1}\right)$, and ordered carbonyl groups $\left(1696 \mathrm{~cm}^{-1}\right)$, respectively

the ESI mass spectrum of BIBSCPS-S3-Ar heated at $80{ }^{\circ} \mathrm{C}$ for $24 \mathrm{~h}$ in DMF under an air atmosphere exhibits the corresponding disulfide (BIBSCPS-S2-Ar) and tetrasulfide (BIBSCPSS4-Ar) analogues. From this observation, it has become clear that the trisulfide linkage of BIBSCPS-S3-Ar behaves as a DCB to afford air-stable dithiyl and thiyl radicals like BITEMPS-S3-Ar.

Subsequently, the difference in hydrogen bonding behavior among the urethane linkages of the BITEMPS-S3 and BIBSCPSS3 moieties was investigated using FT-IR spectroscopy (Fig. 2). For BITEMPS-S3-Ar, there could be seen a well-defined absorption peak with maximum at $1696 \mathrm{~cm}^{-1}$ corresponding to ordered hydrogen-bonded $-\mathrm{C}=\mathrm{O}$ stretching and two shoulders at 1715 and $1732 \mathrm{~cm}^{-1}$ attributed to disordered hydrogenbonded and free $-\mathrm{C}=\mathrm{O}$ stretching, respectively. ${ }^{27}$ On the other hand, BIBSCPS-S3-Ar shows a well-defined peak with maximum at $1715 \mathrm{~cm}^{-1}$ with a shoulder around $1696 \mathrm{~cm}^{-1}$. Moreover, there could be seen a more obvious peak at $1732 \mathrm{~cm}^{-1}$ in BIBSCPS-S3-Ar compared to BITEMPS-S3-Ar. For BITEMPS-S3diacrylate, two characteristic peaks at 1715 and $1692 \mathrm{~cm}^{-1}$ were observed, while BIBSCPS-S3-diacrylate showed a broad peak with maximum at $1715 \mathrm{~cm}^{-1}$. Besides, BIBSCPS-S3 $-{ }^{n}$ Bu showed a little broader peak from 1740 to $1670 \mathrm{~cm}^{-1}$ with maximum at $1692 \mathrm{~cm}^{-1}$ compared to BITEMPS-S3- ${ }^{n} \mathrm{Bu}$. These observations indicate that hydrogen bonding among urethane linkages is partially disrupted by the bulky spirocyclohexyl-substituent groups on the piperidine rings. Also, the degree of the effect of the spirocyclohexyl-substituent groups on hydrogen bonding behavior strongly depends on the molecular structure.

The spirocyclohexyl-substitution also affected chemical properties such as melting point $\left(T_{\mathrm{m}}\right)$. As shown in Table S2 (ESI $\dagger)$, the $T_{\mathrm{m}}$ values of BIBSCPS-S3-Ar $\left(220.4{ }^{\circ} \mathrm{C}\right)$ and BIBSCPS$\mathrm{S} 3-{ }^{n} \mathrm{Bu}\left(75.0{ }^{\circ} \mathrm{C}\right)$ are higher than those of the corresponding BITEMPS-S3 compounds [BITEMPS-S3-Ar $\left(153.7{ }^{\circ} \mathrm{C}\right)$ and BITEMPS-S3 $\left.-{ }^{n} \mathrm{Bu}\left(55.5{ }^{\circ} \mathrm{C}\right)\right]$, in spite of the partial disruption of hydrogen bonding induced by the spirocyclohexyl substituent groups. This is probably because the molecular mobility is highly suppressed by bulky spirocyclohexyl-substituent groups.

\section{Evaluation of polymethacrylate networks}

Cross-linked polymers containing the BITEMPS-S3 or BIBSCPSS3 moieties at the cross-linking points were prepared. As shown in Scheme 3, BITEMPS-S3-diacrylate and BIBSCPS-S3-diacrylate were employed as dynamic cross-linkers and incorporated into polymer networks via radical polymerization using various

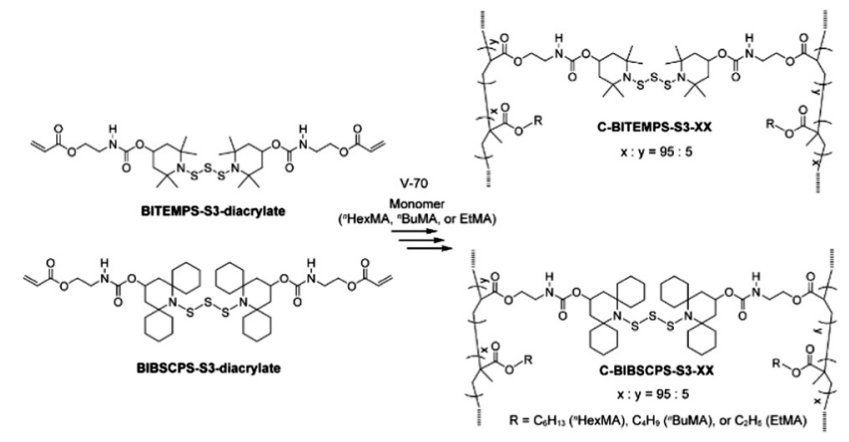

Scheme 3 Synthesis of cross-linked polymers containing the BIBSCPSS3 or BITEMS-S3 moieties.

methacrylate monomers (ethyl (EtMA), butyl ( ${ }^{n}$ BuMA), or hexyl $\left({ }^{n}\right.$ HexMA) methacrylates) and azo-based low-temperature radical initiator V-70. The concentration of the cross-linker was set to $5 \mathrm{~mol} \%$ with respect to the total amount of monomer and crosslinkers. The cross-linked polymers were washed several times with chloroform/methanol to remove any unreacted chemicals and dried in a vacuum at $50{ }^{\circ} \mathrm{C}$ for $24 \mathrm{~h}$ before characterization. The prepared cross-linked polymers are referred to as C-BITEMPS-S3-XX or C-BIBSCPS-S3-XX, where XX indicates the matrix polymer (EtMA, ${ }^{n}$ BuMA, or ${ }^{n}$ HexMA). All of the obtained cross-linked polymers were transparent as shown in Fig. S1 and S2 (ESI $\dagger$ ). Additionally, there could not be observed the differences in thermal properties such as the $5 \%$ weight loss $\left(T_{\mathrm{d} 5}\right)$ and glass transition $\left(T_{\mathrm{g}}\right)$ temperatures for BITEMPS-S3 and BIBSCPS-S3 in the network system (Table S3, ESI $\dagger$ ).

In order to evaluate the differences in thermal exchangeability of the BITEMPS-S3 and BIBSCPS-S3 moieties in the polymer networks, temperature-dependent stress-relaxation experiments were carried out because the topology rearrangement of the polymer networks can occur through thermal exchangeable reactions. A 3\% elongation strain was applied on the samples at different temperatures (from 110 to $140{ }^{\circ} \mathrm{C}$ ) and the relaxation modulus was measured as a function of time. As can be seen in Fig. 3, the stress-relaxation behaviors for all of the cross-linked polymers were clearly observed, indicating that the bond-exchange reaction among the BITEMPS-S3 or BIBSCPS-S3 moieties occurred in the bulk materials. Meanwhile, the normalized relaxation modulus at time $t=0\left(\sigma / \sigma_{0}\right)$ could not be completely relaxed and became equilibrated within $10-60 \mathrm{~min}$ at $120-140{ }^{\circ} \mathrm{C}$, indicating that the chaintransfer reaction at the BITEMPS-S3 and BIBSCPS-S3 moieties during radical polymerization led to the formation of a nondynamic cross-linking point. Weibul and KohlrauschWilliams-Watts function, which accounts for a breadth of relaxation time, is used to describe stress relaxation in polymers and expressed as the following equation: ${ }^{28}$

$$
\frac{\sigma}{\sigma_{0}}=\exp \left\{-\left(\frac{t}{\tau^{*}}\right)^{\beta}\right\}+\frac{\sigma_{\text {perm }}}{\sigma_{0}}
$$

where $\beta(0<\beta \leq 1)$ is the stretching exponent which serves as a shape parameter characterizing the breadth of the relaxation distribution, $\tau^{*}$ is the characteristic relaxation time, and 

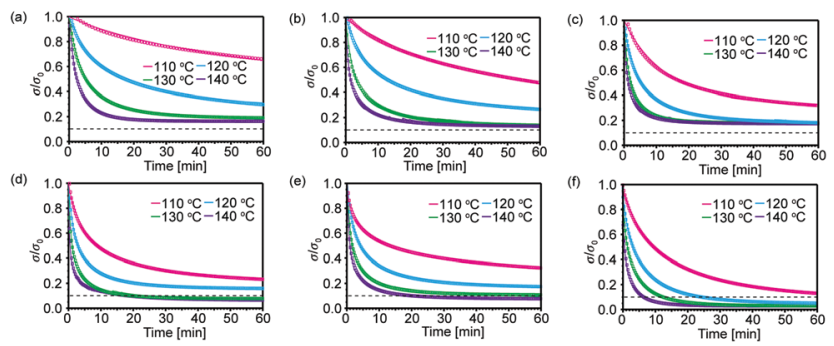

Fig. 3 Stress relaxation curves of (a) BITEMPS-S3-EtMA, (b) BITEMPSS3- ${ }^{n}$ BuMA, (c) BITEMPS-S3- ${ }^{n}$ HexMA, (d) BIBSCPS-S3-EtMA, (e) BIBSCPSS3- ${ }^{n}$ BuMA, and (f) BIBSCPS-S3- ${ }^{n}$ HexMA, respectively, at different temperatures. The dotted lines indicate $\sigma / \sigma_{0}=0.1$. The solid lines are original experimental data, fitted by the stretched exponential model (shown as open squares).

$\sigma_{\text {perm }} / \sigma_{0}$ is the residual relaxation modulus at $t=\infty$. Also, the average relaxation time, $\langle\tau\rangle$, is calculated using the following equation:

$$
\langle\tau\rangle=\frac{\tau^{*} \Gamma(1 / \beta)}{\beta}
$$

where $\Gamma$ represents the gamma function. We applied eqn (1) for fitting the stress-relaxation curves and summarized the fitting parameters $\left(\tau^{*}, \beta\right.$, and $\left.\sigma_{\text {perm }} / \sigma_{0}\right)$ and calculated the average relaxation time $(\langle\tau\rangle)$ from eqn (2) in Table 1 and Table S4 (ESI $\dagger$ ). As shown in Table S4 (ESI $\dagger$ ), the $\langle\tau\rangle$ values decrease with increase in temperature, implying a change in polymer network structure as a result of more bond-exchange reaction occur at higher temperature. Furthermore, the activation energy $\left(E_{\mathrm{a}}\right)$ was estimated from the slope of the obtained $\ln \langle\tau\rangle$ versus 1/temperature plot (Fig. S7, ESI $\dagger$ ). As shown in Table 1, CBITEMPS-S3-XX exhibits higher $E_{\mathrm{a}}$ values (21.4-31.8 $\mathrm{kcal} \mathrm{mol}^{-1}$ ) than C-BIBSCPS-S3-XX (12.1-18.0 $\mathrm{kcal} \mathrm{mol}^{-1}$ ), indicating that the modification to spirocyclohexyl groups in a piperidine ring increases the steric hindrance effect and enhances thermal exchangeability in the polymer network. Moreover, it should be noted that the $\sigma_{\text {perm }} / \sigma_{0}$ values of C-BITEMPS-S3-XX (0.12-0.17) are higher than those of C-BIBSCPS-S3-XX (0.02-0.07). It was previously revealed that the BITEMPS-S2

Table 1 Thermal properties and parameters based on Weibul and Kohlrausch-Williams-Watts function fitting to stress relaxation experimental data of cross-linked polymers

\begin{tabular}{|c|c|c|c|c|c|c|}
\hline Polymer & $\begin{array}{l}T_{\mathrm{g}}{ }^{a} \\
{\left[{ }^{\circ} \mathrm{C}\right]}\end{array}$ & $\begin{array}{l}\tau^{* b} \\
{[\min ]}\end{array}$ & $\begin{array}{l}\beta^{b} \\
{[-]}\end{array}$ & $\begin{array}{l}\sigma_{\text {perm }} / \sigma_{0}{ }^{b} \\
{[-]}\end{array}$ & $\begin{array}{l}\langle\tau\rangle^{c} \\
{[\min ]}\end{array}$ & $\begin{array}{l}E_{\mathrm{a}}{ }^{d} \\
{\left[\mathrm{kcal} \mathrm{mol}^{-1}\right]}\end{array}$ \\
\hline C-BITEMPS-S3-EtMA & 43.0 & 1.16 & 0.61 & 0.15 & 1.68 & 31.8 \\
\hline C-BITEMPS-S3- ${ }^{n}$ BuMA & 21.9 & 1.65 & 0.51 & 0.12 & 3.12 & 25.1 \\
\hline C-BITEMPS-S3- ${ }^{n}$ HexMA & 11.7 & 1.12 & 0.51 & 0.17 & 2.12 & 21.4 \\
\hline C-BIBSCPS-S3-EtMA & 37.5 & 1.10 & 0.55 & 0.07 & 2.40 & 12.1 \\
\hline C-BIBSCPS-S3- ${ }^{n}$ BuMA & 20.7 & 1.11 & 0.45 & 0.07 & 2.69 & 15.9 \\
\hline C-BIBSCPS-S3- ${ }^{n}$ HexMA & 12.4 & 1.15 & 0.51 & 0.02 & 2.21 & 18.0 \\
\hline
\end{tabular}

${ }^{a} T_{\mathrm{g}}$ was determined from DSC measurement at a heating rate of $20{ }^{\circ} \mathrm{C}$ $\min ^{-1}$ under nitrogen atmosphere. ${ }^{b} \tau^{*}, \beta$, and $\sigma_{\text {perm }} / \sigma_{0}$ values were estimated from Weibul and Kohlrausch-Williams-Watts function to a stress-relaxation curve measured at $140{ }^{\circ} \mathrm{C}$ using eqn (1). ${ }^{c}\langle\tau\rangle$ was calculated from eqn (2). ${ }^{d} E_{\mathrm{a}}$ was estimated from Arrhenius plot of $\ln \langle\tau\rangle$ vs. $1 /$ temperature.
Table 2 Chain-transfer constants of BITEMPS-S3- ${ }^{n} \mathrm{Bu}$ and BIBSCPSS3- ${ }^{n} \mathrm{Bu}^{a}$

\begin{tabular}{llll}
\hline & \multicolumn{1}{c}{$C_{\mathrm{tr}}{ }^{b}$} & & \\
\cline { 2 - 4 } CTA & EtMA & ${ }^{n}$ BuMA & ${ }^{n}$ HexMA \\
\hline BITEMPS-S3- ${ }^{n} \mathrm{Bu}$ & 2.74 & 1.01 & 1.38 \\
BIBSCPS-S3- ${ }^{n} \mathrm{Bu}$ & 0.84 & 0.25 & 0.30
\end{tabular}

${ }^{a}$ Radical polymerization was carried out in DMAc at $30^{\circ} \mathrm{C}$ for $2 \mathrm{~h}$ using $\mathrm{V}-70$ as an initiator in the presence of BITEMPS-S3- ${ }^{n} \mathrm{Bu}$ or BIBSCPSS3 ${ }^{n}$ Bu. [initiator] : [monomer] was $1: 100 .{ }^{b} C_{\mathrm{tr}}$ was calculated from Mayo's equation.

moiety incorporated into poly $\left({ }^{n} \mathrm{HexMA}\right)$ at a cross-linking point shows lower $\sigma / \sigma_{0}$ value than BITEMPS-S3, probably due to higher chain-transfer constant $\left(C_{\mathrm{tr}}\right)$ of the trisulfide linkage compared to the disulfide linkage. ${ }^{17,29}$ Considering that the oxidative stability of the 2,2,6,6-tetramethylpiperidine- $N$-oxyl (TEMPO) to ascorbic acid was increased by replacing the methyl groups in TEMPO with spirocyclohexyl groups due to effective steric shielding, ${ }^{30}$ this considerable difference would indicate that the chain-transfer reaction to S-S linkage in the BIBSCPSS3 moiety during radical polymerization is highly suppressed by the steric hindrance of the bulky spirocyclohexyl groups.

To support this assumption, $C_{\text {tr }}$ of EtMA, ${ }^{n}$ BuMA, and ${ }^{n}$ HexMA for BITEMPS-S3 and BIBSCPS-S3 moieties were determined by the Mayo method using BITEMPS-S3 $-{ }^{n} \mathrm{Bu}$ and BIBSCPS-S3- ${ }^{n} \mathrm{Bu}$ as chaintransfer agents (CTAs). ${ }^{31}$ The results are summarized in Table 2 (see also Table S5 and Fig. S8, ESI $\dagger$ ). As expected, BITEMPS-S3- ${ }^{n} \mathrm{Bu}$ exhibits approximately 4 times higher $C_{\mathrm{tr}}$ values than BIBSCPSS3- ${ }^{n} \mathrm{Bu}$ regardless of alkyl chain length in methacrylate monomers. These results are in good accordance with the $\sigma_{\text {perm }} / \sigma_{0}$ values of stress-relaxation experiments. Therefore, it became clear that BIBSCPS-S3-diacrylate was more efficiently reacted without chaintransfer reaction via radical polymerization to produce cross-linked poly(methacrylate) compared to the BITEMPS-S3 moiety.

Then, cyclic tensile tests were carried out with various maximum strain in order to investigate the differences in energy dissipation caused by DCBs. As shown in Fig. 4, hysteresis loops of both C-BIBSCPS-S $3{ }^{n}{ }^{n}$ HexMA and C-BITEMPSS3- ${ }^{n}$ HexMA are enhanced with increasing strain, which indicates that the efficient stress dissipation occurred in the BITEMPS-S3 and BIBSCPS-S3 moieties. In addition, the dependence of applied energy on residual strain was also compared, since there could be seen differences in the Young's modulus
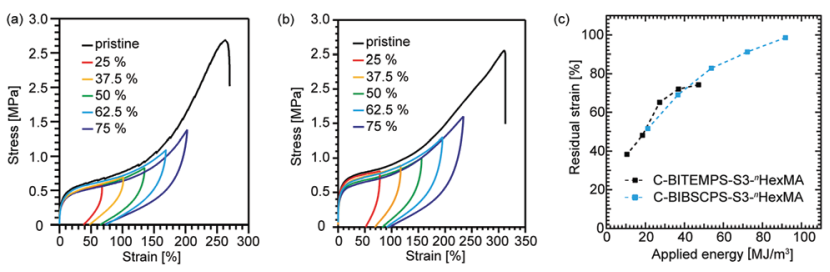

Fig. 4 Cyclic stress-strain curves of (a) C-BITEMPS-S3- ${ }^{n}$ HexMA and (b) C-BIBSCPS-S3- ${ }^{n}$ HexMA under different maximum strains ranged from $25 \%$ to $75 \%$ toward each fracture strain. (c) Relationship between applied energy and residual strain. 
and fracture strain of C-BIBSCPS-S $3-{ }^{n}$ HexMA and C-BITEMPSS3- ${ }^{n}$ HexMA. As exhibited in Fig. 4c, it became apparent that C-BIBSCPS-S3- ${ }^{n}$ HexMA showed almost the same residual strain value of C-BITEMPS-S3- ${ }^{n}$ HexMA, when the same elongation energies were applied. Although there could be seen differences in the $\sigma_{\text {perm }} / \sigma_{0}$ and $C_{\text {tr }}$ values, the energy-dissipation behaviors of these cross-linked polymers slightly resemble each other, implying that the non-dynamic cross-linking point resulted from a chain-transfer reaction to the trisulfide linkage would little affect the energy-dissipating mechanism. Furthermore, this observation also indicates that the recombination rates of the cleaved trisulfide linkages in the BIBSCPS-S3 moiety would be almost equal to that in the BITEMPS-S3 ones, in spite of the large steric hindrance of spirocyclohexyl groups.

\section{Thermal healing property}

Finally, the self-healing properties of C-BITEMPS-S3- ${ }^{n}$ HexMA and C-BIBSCPS-S3- ${ }^{n}$ HexMA were quantitatively evaluated using the tensile tests with dogbone-shaped specimens punched out from polymer films. After each dogbone-shaped specimen to be healed was cut into two pieces at the center with a blade, the cut surfaces were then in contact, tightly sandwiched between stainless plates by two paper clips, and healed at various temperatures for $24 \mathrm{~h}$. Results of tensile tests are summarized in Table S7 (ESI $\dagger$ ). As shown in Fig. 5, both C-BITEMPSS3- ${ }^{n}$ HexMA and C-BIBSCPS-S3- ${ }^{n}$ HexMA healed at $110{ }^{\circ} \mathrm{C}$ exhibit almost comparable mechanical properties to the pristine state. The healing efficiency of C-BITEMPS-S3- ${ }^{n}$ HexMA decreases below $100{ }^{\circ} \mathrm{C}$ and C-BITEMPS-S3- ${ }^{n}$ HexMA healed at $90{ }^{\circ} \mathrm{C}$ apparently fractured along with the cut line (Fig. 5b). Meanwhile, the healability of the mechanical properties for C-BIBSCPS-S3- ${ }^{n}$ HexMA healed even at $90{ }^{\circ} \mathrm{C}$ is $95 \%$ and $96 \%$ for the fracture strain and maximum. C-BITEMPS-S $3-{ }^{n}$ HexMA healed at $90{ }^{\circ} \mathrm{C}$ fractured near the edge or cut line, which are different from the cut lines, and supports that the mechanical properties of the healed part are equivalent to the pristine state (Fig. 5c). However, healability of C-BIBSCPS-S3 $-{ }^{n}$ HexMA drops sharply to $63 \%$ and $62 \%$ for the fracture strain and maximum, when healed at $80{ }^{\circ} \mathrm{C}$. Considering almost the same $T_{\mathrm{g}}$ of C-BITEMPS-S3- ${ }^{n}$ HexMA and C-BIBSCPS-S3- ${ }^{n}$ HexMA, the enhancement in healing efficiency of C-BIBSCPS-S $3-{ }^{n}$ HexMA would be
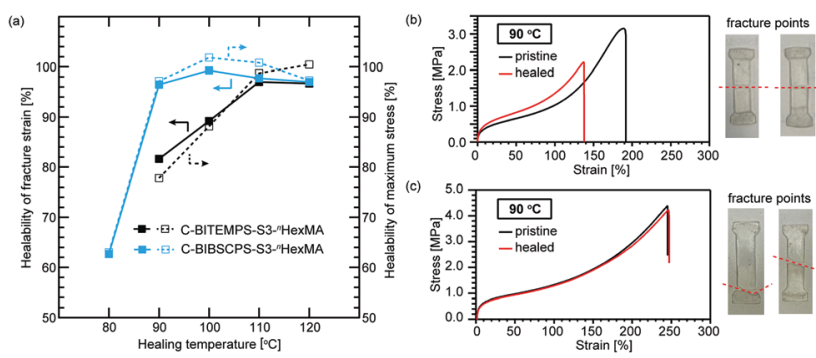

Fig. 5 (a) Healability of fracture strain and maximum stress of C-BITEMPS-S3- ${ }^{n}$ HexMA and C-BIBSCPS-S3- ${ }^{n}$ HexMA after heating at different temperatures for $24 \mathrm{~h}$. Stress-strain curves and fracture points of (b) C-BITEMPS-S3- ${ }^{n}$ HexMA and (c) C-BIBSCPS-S3- ${ }^{n}$ HexMA healed at $90{ }^{\circ} \mathrm{C}$ for $24 \mathrm{~h}$. owing to relatively high thermal exchangeability in bulk as well as the non-dynamic cross-linking point resulted from its lower $C_{\text {tr }}$ value for ${ }^{n}$ HexMA. In other words, the steric hindrance induced by the bulky spirocyclohexyl groups in the BIBSCPS-S3 moiety not only promoted thermal exchangeability but also suppressed chain-transfer reaction for trisulfide linkage during the polymerization, so that the pristine BIBSCPS-S3 moiety could be efficiently incorporated into the cross-linking point via radical polymerization.

\section{Conclusions}

In summary, we have designed and synthesized a highly sterically hindered dialkylamino trisulfide (BIBSCPS-S3). Structural analysis by single-crystal X-ray diffraction revealed that all spirocyclohexyl groups are away from the $\mathrm{N}-\mathrm{S}$ bonds because of large steric repulsion between sulfur and cyclohexyl hydrogen atoms. Estimation of the chain-transfer constants indicated that these bulky spirocyclohexyl groups in BIBSCPS-S3 suppressed the chain-transfer reaction to the trisulfide linkage during radical polymerization. In bulk, stress-relaxation experiments revealed that the increase in steric hindrance could not only decrease the activation energy for thermal exchange reaction in bulk but also suppress chain-transfer reaction during radical polymerization to some extent. Therefore, cross-linking points containing a BIBSCPS-S3 moiety were efficiently incorporated into the polymer networks with ethyl, $n$-butyl, or $n$-hexyl methacrylate monomers. As a result, BIBSCPS-S3cross-linked poly( $n$-hexyl methacrylate) realized nearly quantitative damage healability only by simple hot pressing at $90{ }^{\circ} \mathrm{C}$ under mild pressure for $24 \mathrm{~h}$. Although BIBSCPS-S3 turned out to be one of the DCBs triggered upon exposure to heating at lower temperature than BITEMPS-S3, a detailed study on its disulfide analogue (BIBSCPS-S2) will be required to enhance the steric hindrance effect of spirocyclohexyl groups.

\section{Author contributions}

M. A., C. O., A. T., and H. O. designed the research plan. M. A. performed the molecular synthesis. M. A., M. Y., M. F., K. O., Y. I., and M. O. performed characterization. T. K. performed $\mathrm{X}$-ray single crystal analysis. M. A. drafted the manuscript, and C. O., A. T., and H. O. critically reviewed and revised the final manuscript. All authors commented on the manuscript and agree with the results and conclusions.

\section{Conflicts of interest}

There are no conflicts to declare.

\section{Acknowledgements}

This research was financially supported by The Naito Science \& Engineering Foundation. The authors appreciate the helpful 
discussion of Dr Kazunori Nakano, Nagoya Municipal Industrial Research Institute.

\section{Notes and references}

1 H. Otsuka, Polym. J., 2013, 45, 879-891.

2 P. Berto, A. Pointet, C. Le Coz, S. Grelier and F. Peruch, Macromolecules, 2018, 51, 651-659.

3 R. Snyder, D. J. Fortman, G. X. De Hoe, M. A. Hillmyer and W. R. Dichtel, Macromolecules, 2018, 51, 389-397.

4 J. A. Yoon, J. Kamada, K. Koynov, J. Mohin, R. Nicolaÿ, Y. Zhang, A. C. Balazs, T. Kowalewski and K. Matyjaszewski, Macromolecules, 2012, 45, 142-149.

5 X. Luo and P. T. Mather, Macromolecules, 2010, 6, 2146.

6 D. Y. Wu, S. Meure and D. Solomon, Prog. Polym. Sci., 2008, 33, 479-522.

7 C. Yuan, M. Z. Rong, M. Q. Zhang, Z. P. Zhang and Y. C. Yuan, Chem. Mater., 2011, 23, 5076-5081.

8 M. A. Bin Rusayyis and J. M. Torkelson, Polym. Chem., 2021, 12, 2760-2771.

9 A. Takahashi, R. Goseki and H. Otsuka, Angew. Chem., Int. Ed., 2017, 56, 2016-2021.

10 H. Yokochi, R. Takashima, D. Aoki and H. Otsuka, Polym. Chem., 2020, 11, 3557-3563.

11 A. Tsuruoka, A. Takahashi, D. Aoki and H. Otsuka, Angew. Chem., Int. Ed., 2020, 59, 4294-4298.

12 N. Tsurumi, R. Takashima, D. Aoki, S. Kuwata and H. Otsuka, Angew. Chem., Int. Ed., 2020, 59, 4269-4273.

13 A. Takahashi, R. Goseki, K. Ito and H. Otsuka, ACS Macro Lett., 2017, 6, 1280-1284.

14 R. Takashima, D. Aoki and H. Otsuka, Macromolecules, 2020, 53, 4670-4677.

15 S. Kataoka, A. Tsuruoka, D. Aoki and H. Otsuka, ACS Appl. Polym. Mater., 2021, 3, 888-895.

16 J. Kida, D. Aoki and H. Otsuka, ACS Macro Lett., 2021, 10, 558-563.
17 M. Aiba, T. Koizumi, M. Futamura, K. Okamoto, M. Yamanaka, Y. Ishigaki, M. Oda, C. Ooka, A. Tsuruoka, A. Takahashi and H. Otsuka, ACS Appl. Polym. Mater., 2020, 2, 4054-4061.

18 Y. Jing, A. Mardyukov, K. Bergander, C. G. Daniliuc and A. Studer, Macromolecules, 2014, 47, 3595-3602.

19 Y. Miura, A. Ichikawa and I. Taniguchi, Polymer, 2003, 44, 5187-5194.

20 Y. Miura, N. Nakamura and I. Taniguchi, Macromolecules, 2001, 34, 447-455.

21 I. A. Kirilyuk, Y. F. Polienko, O. A. Krumkacheva, R. K. Strizhakov, Y. V. Gatilov and I. A. Grigor'ev, J. Org. Chem., 2012, 77, 8016-8027.

22 T. Yamasaki, F. Mito, Y. Ito, S. Pandian, Y. Kinoshita, K. Nakano, R. Murugesan, K. Sakai, H. Utsumi and K. I. Yamada, J. Org. Chem., 2011, 76, 435-440.

23 S. Morisako, R. Shang and Y. Yamamoto, Inorg. Chem., 2016, 55, 10767-10773.

24 K. Sakai, K. Yamada, T. Yamasaki, Y. Kinoshita, F. Mito and H. Utsumi, Tetrahedron, 2010, 66, 2311-2315.

25 G. Stevanato, G. Casano, D. J. Kubicki, Y. Rao, L. E. Hofer, G. Menzildjian, H. Karoui, D. Siri, M. Cordova, M. Yulikov, G. Jeschke, M. Lelli, A. Lesage, O. Ouari and L. Emsley, J. Am. Chem. Soc., 2020, 142, 16587-16599.

26 Z. Ma, Q. Huang and J. M. Bobbitt, J. Org. Chem., 1993, 58, 4837-4843.

27 S. Shetranjiwalla, S. Li, L. Bouzidi and S. S. Narine, J. Renewable Mater., 2017, 5, 333-344.

28 K. S. Fancey, J. Mater. Sci., 2005, 40, 4827-4831.

29 W. C. Danen and D. D. Newkirk, J. Am. Chem. Soc., 1976, 516, 516-520.

30 M. M. Haugland, J. E. Lovett and E. A. Anderson, Chem. Soc. Rev., 2018, 47, 668-680.

31 D. M. Haddleton, D. R. Maloney, K. G. Suddaby, A. V. G. Muir and S. N. Richards, Macromol. Symp., 1996, 111, 37-46. 